\title{
What is the best initial therapy for the average patient with idiopathic achalasia?
}

\author{
Eric Semlacher MD FRCPC
}

\section{ARTICLE}

Finley RJ, Clifton JC, Stewart KC, Graham AJ, Worsley DF. Laparoscopic Heller myotomy improves esophageal emptying and the symptoms of achalasia. Archives of Surgery 2001;136:892-6. tion in the other seven patients were not identified, and while these patients were said to have similar improvement in symptom relief and esophageal transit compared with those who underwent anterior fundoplication, specific long term follow-up was not described.

Eighty-nine patients were available for postdischarge follow-up, with a median follow-up of 18 months. The reasons for dropouts and the short term outcomes for patients not included in the follow-up group were not disclosed. Only one patient continued to have regurgitation more than once a week after surgery. Two patients continued to have significant dysphagia postoperatively. Both symptoms had been prominent in nearly all patients preoperatively. One of the dysphagic patients required laparoscopic takedown of the fundoplication, while the other responded to balloon dilation of the distal esophagus. Preoperative and postoperative standardized scintigraphic esophageal transit studies were obtained in 67 patients. There was statistically significant improvement in esophageal transit in both the early and the late postoperative follow-up studies. Eighty-seven of 89 patients reported being very satisfied with the surgical outcome. One patient was somewhat satisfied, and one was not at all satisfied.

\section{COMMENTARY}

Etiology-oriented therapy for idiopathic achalasia does not yet exist. Consequently, current treatment continues to focus on relieving the functional obstruction that the disease produces at the level of the LES. The most satisfactory 
way of achieving this in the average patient with achalasia remains a matter of opinion.

Pharmacological therapies have met with very limited success. Injection of botulinum toxin into the LES region had shown promise in early reports, with initial symptom improvement noted in up to $60 \%$ to $75 \%$. However, the benefit of this treatment tends to be quite short lived in the majority of cases. Moreover, similar to pharmacological treatment, botulinum toxin has been shown to have a greater effect on symptoms than on LES pressure and esophageal emptying. Physical disruption of the LES has been the mainstay of treatment for decades. This has been achieved in the past, either by forceful pneumatic dilation using a balloon in the esophageal lumen or by surgical myotomy. When successful, either approach can provide symptomatic relief and improved esophageal emptying.

Although LES disruption can be achieved in a more controlled and precise manner at surgery, pneumatic dilation has been favoured historically as first-line therapy. This is because the rates of both success and complications have been considered entirely acceptable for pneumatic dilation in experienced hands, when faced with the alternative of a surgical procedure that was commonly associated with significant discomfort and relatively long postoperative recovery periods. Surgery was reserved commonly for complications or failures of pneumatic dilation. However, the time has come to re-evaluate that position.

Minimally invasive approaches to esophageal myotomy have dominated the surgical literature over the past decade. The present report describes the extensive experience of one Canadian surgeon (RJ Finley) with these techniques. Like many others, Finley has come to favour the laparoscopic (abdominal) Heller myotomy over a thoracoscopic approach, and the paper reports his results during the first six years after he switched to that technique in 1994. Partial fundoplication, done in conjunction with the laparoscopic myotomy, is performed because the degree of mobilization at the esophagogastric junction resulting from surgery increases the likelihood of subsequent pathological reflux. The initial and medium term follow-up results reported by Finley et al are quite typical of those reported from other high quality centres around the world - following an initial learning curve for the surgeon, results are good to excellent in $90 \%$ or more of patients. Moderate followups (in the range of two years) in this and other case series have demonstrated lasting symptom control and patient satisfaction with the procedure. A small number of the follow-ups from the present study have shown good results, extending to six years. Whether these good results will persist for more extended periods remains to be seen. Where long term response data are available in the literature, there has been a higher rate of unsatisfactory treatment than was previously appreciated with both pneumatic dilation and myotomy (1). Because a significant contributor to long term dissatisfaction with all of the LES-disrupting interventions (surgical or otherwise) has been the development of late gastroesophageal reflux complications such as stricture formation, it is hoped that routine use of fundoplication will improve these long term outcomes.

So, what might the standard of care for idiopathic achalasia look like in the near future? As more centres boast surgeons who are well trained in this technique, there seems to be little doubt that laparoscopic myotomy will become the treatment of first choice for the average patient with achalasia. The minimally invasive myotomy procedures are as effective as their open counterparts, yet they have the advantages of shorter hospital stays, less time absent from work, less blood loss during surgery and less incision-related morbidity. The champions of pneumatic dilation will be hard pressed to prove that their technique can match current surgical intervention, although there will undoubtedly always be individuals or circumstances that will favour trying pneumatic dilation first. An argument might be made for consideration of LES injection with botulinum to provide temporary symptom relief while the patient awaits surgical intervention. Attractive features of this approach are its relative noninvasiveness, relatively high initial response rate and ease of access for patients because injection therapy might conceivably be offered in almost any community having endoscopic capability. However, the benefit of this approach would need to be weighed against the increasing evidence that botulinum protein injection can induce inflammation and fibrosis in the esophageal wall that may make subsequent surgical therapy more difficult. Pharmacological therapy should be considered in each case and, for those few individuals who find it to be effective and reasonably well tolerated, this could certainly be maintained until more definitive treatment is arranged. Neither of these therapies should be considered substitutes for, nor should they unduly delay, arrangement of more definitive treatment.

A few patients will ultimately continue to have significant problems, despite the available therapies. The existence of this thankfully small population, for whom management can prove to be extremely challenging, should not detract from the significant advances being made in relieving the symptoms of the majority of achalasia sufferers. It does highlight, however, that current treatments represent symptom palliation and that the future focus of therapy will ultimately still need to be aimed at identifying, and finding treatments for, the root cause of idiopathic achalasia.

\section{REFERENCE}

1. Torbey CF, Achkar E, Rice TW, Baker M, Richter JE. Long-term outcome of achalasia treatment: the need for closer follow-up. J Clin Gastroenterol 1999;28:125-30. 


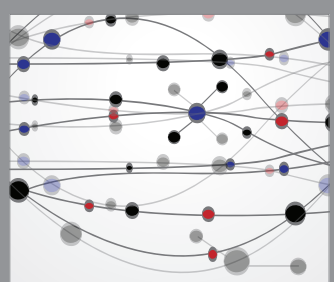

The Scientific World Journal
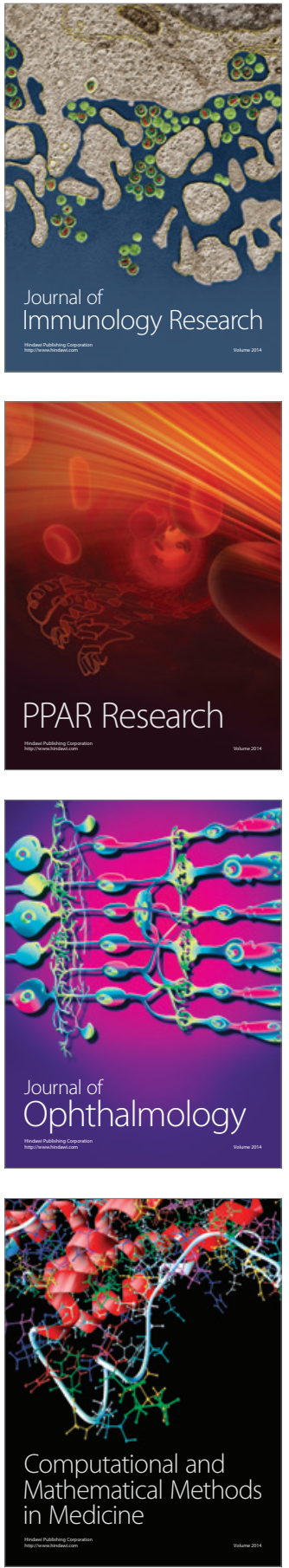

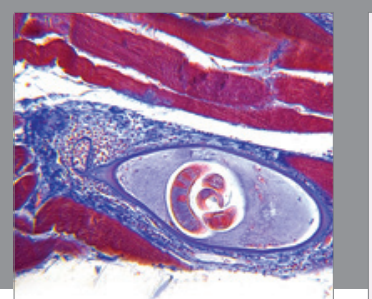

Gastroenterology Research and Practice

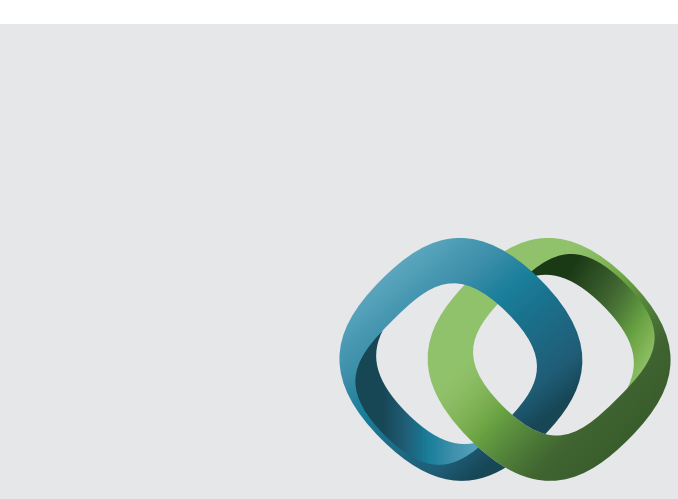

\section{Hindawi}

Submit your manuscripts at

http://www.hindawi.com
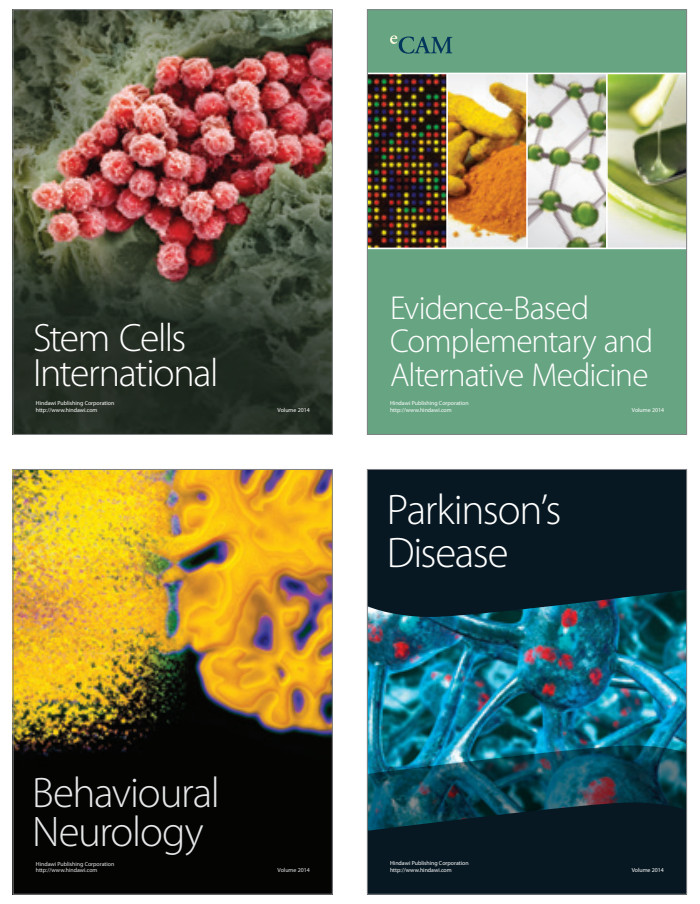
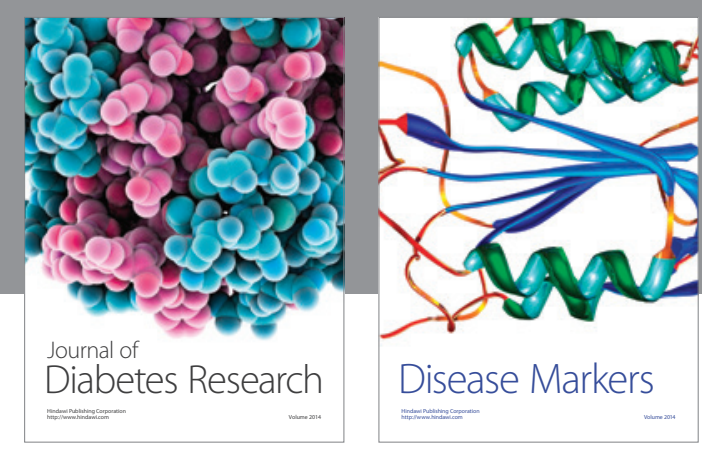

Disease Markers
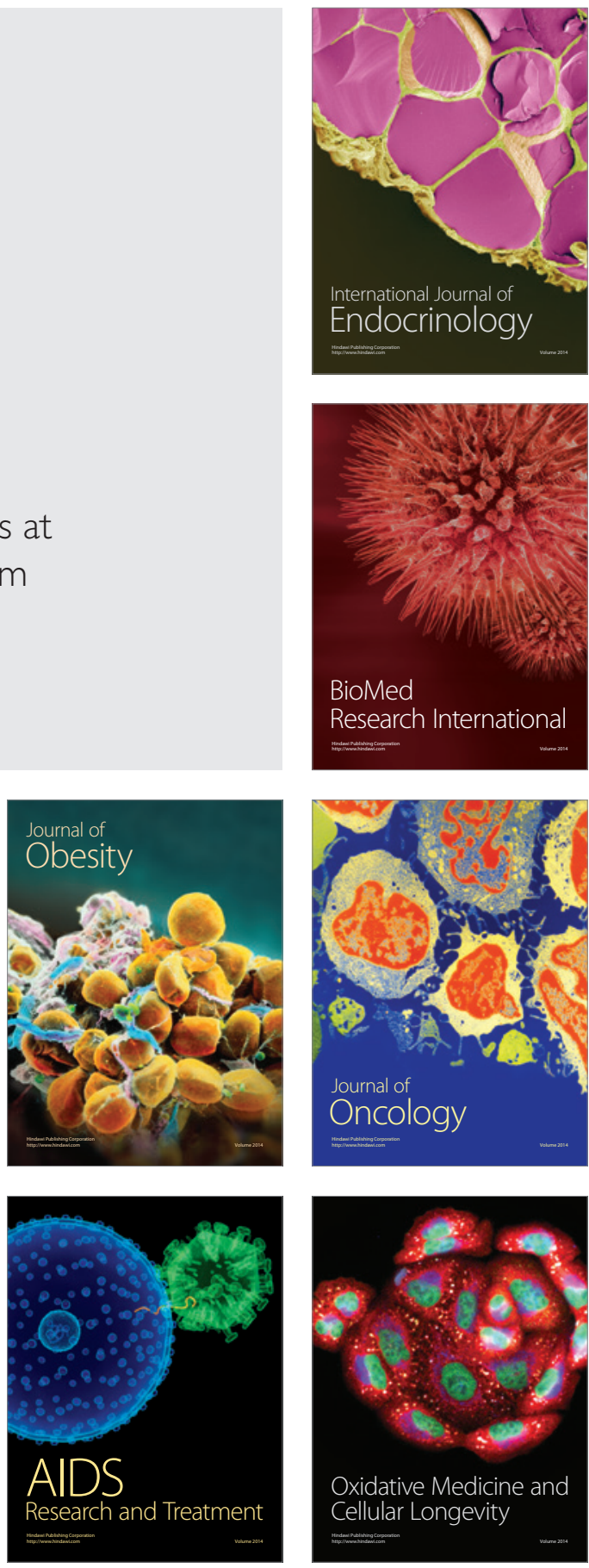